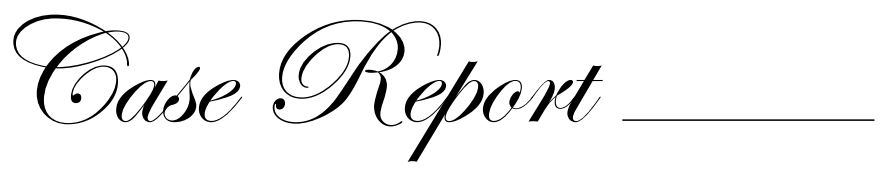

\section{Primary Pancreatic lymphoma -} a rare entity

\section{Introduction}

Primary Pancreatic Lymphoma (PPL) is an extremely rare disease and accounts for only $2 \%$ of extra-nodal lymphomas and $0.5 \%$ of all cases of pancreatic masses ${ }^{1}$. Most of the cases are intermediate or high grade nonHodgkin's Lymphoma, with diffuse large B cell type being the predominant histological type ${ }^{2,3}$. Clinically, PPL is most commonly misdiagnosed as pancreatic cancer. However, unlike carcinoma, PPLs are potentially treatable with chemotherapy and radiotherapy. Using combined modality treatment, cure rates of upto $30 \%$ are reported for patients with PPL which is much better than the dismal 5-year survival rate of $5 \%$ in patients with pancreatic carcinoma ${ }^{4-6}$.

\section{Case}

A 32-year old female presented with a history of vague abdominal pain for two months along with jaundice and decreased appetite for one month. There was no history of altered bowel habit or vomiting. On physical examination, the patient was icteric and mild tenderness was present in the epigastric region. Laboratory tests showed normal blood count. Serum bilirubin (total) was $10.20 \mathrm{mg} / \mathrm{dl}$, serum bilirubin (direct) 6.70 , total protein $7.60 \mathrm{gm} / \mathrm{dl}, \mathrm{A} / \mathrm{G}$ ratio 0.55 , SGOT $162 \mathrm{IU} / \mathrm{L}$, SGPT 152 IU/L, serum alkaline phosphatase 1078 IU and GGT 332 IU/L. Carbohydrate antigen CA 19-9 was raised. Contrast Enhanced Computed Tomography (CECT) of the whole abdomen revealed an ill-defined hypo-dense mass $(5 \mathrm{X} 4 \mathrm{~cm})$ in the uncinate process of the pancreas with infiltration of the second part of the duodenum (Figure 1). Endoscopic ultrasound-guided fine needle aspiration of the pancreatic mass was performed along with biopsies of the duodenal mucosa. Histopathology of the specimen demonstrated large atypical lymphoid cells (Figure 2A) which stained positive for CD 20, CD 10MUM 1 and bcl-6 (Figure $2 \mathrm{~B}, \mathrm{D}-\mathrm{F}$ ) and negative for CD 3(Figure 2C) which was suggestive of high grade non- Hodgkin's lymphoma.
CECT of neck, chest and pelvis were normal. Peripheral blood smear was normal. Bone marrow biopsy did not show any involvement by lymphoma. EUS showed 28X24 mm hypo-echoic mass with well-defined margins seen in the uncinate process of the pancreas. Vascular invasion was not seen. The common bile duct (CBD) and pancreatic duct were dilated and blocked by the mass in the uncinate process. Endoscopic retrograde cholangiopancreatographic (ERCP) stenting was done to relieve obstructive jaundice. Serum bilirubin decreased to $3.2 \mathrm{mg} / \mathrm{dl}$ after 3 weeks of ERCP stenting. The case was discussed in a multidisciplinary clinic and was planned for chemotherapy followed by involved field radiotherapy. She received 6 cycles of R-CHOP (rituximab, cyclophosphamide, adriamycin, vincristine, and prednisolone). She tolerated chemotherapy and had good symptomatic relief. Post-chemotherapy CECT abdomen showed more than 50\% reduction in the mass suggestive of partial response. She was planned for involved-field radiotherapy to a dose of 40Gy/22fractions on Linear Accelerator with 6MV photons. Radiotherapy was well tolerated without any interruptions or major complications.

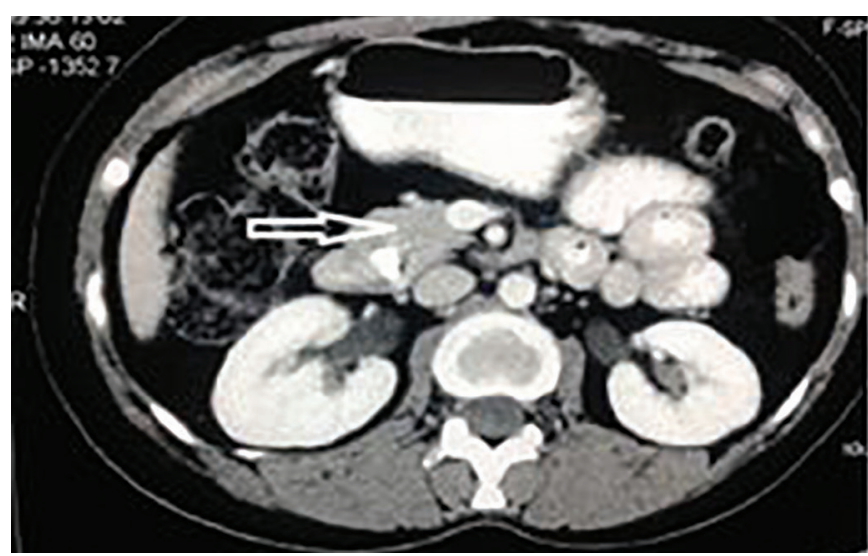

Figure 1: A Contrast Enhanced Computed Tomography Scan of abdomen demonstrating an ill-defined hypodense mass $(5 X 4 \mathrm{~cm})$ in the uncinate process of pancreas with infiltration of second part of duodenum, Common Bile Duct stent in place.

\section{Discussion}

Primary pancreatic lymphoma is a very rare disease ${ }^{1}$. The diagnostic criteria, defined by Dawson et al include the following: mass predominantly within the pancreas with grossly involved lymph nodes confined to the peri-pancreatic region, no palpable superficial 


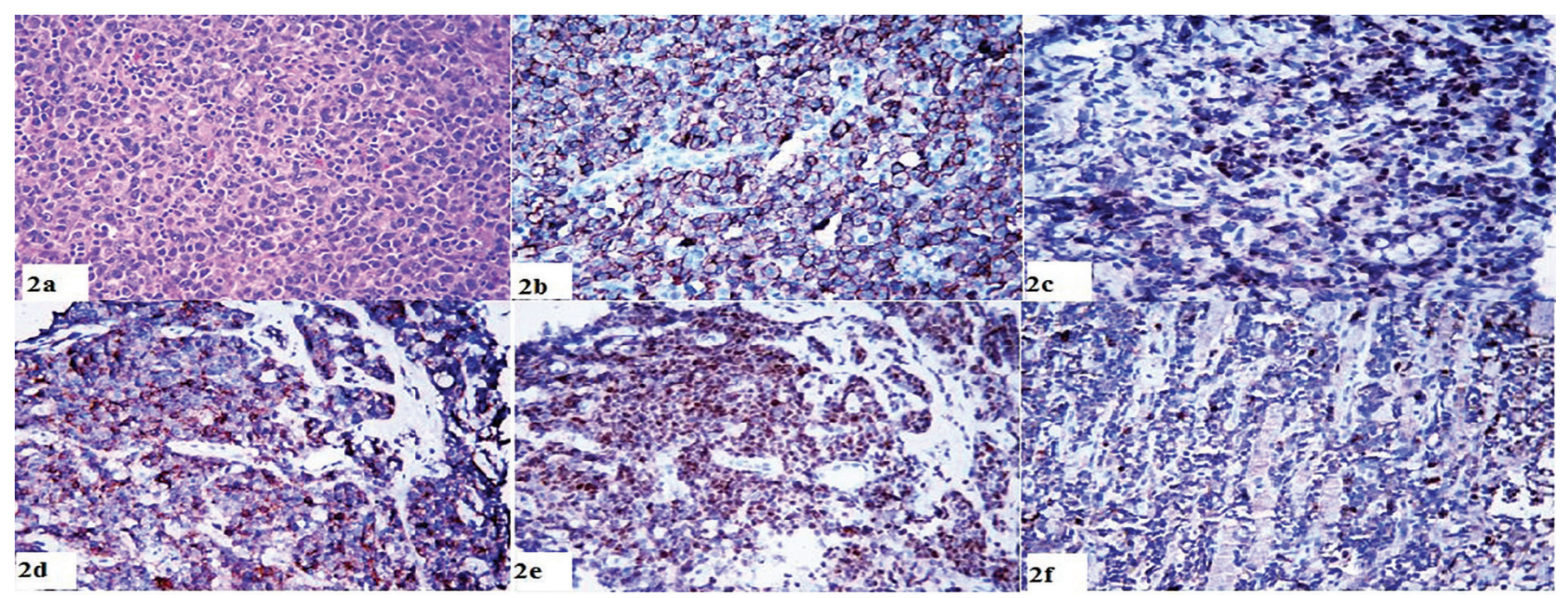

Figure 2 (a-f): High power photomicrograph shows a tumor composed of atypical lymphoid cells. Parent tissue is not identified.These cells have large pleomorphic vesicular nuclei with prominent nucleoli.Atypical mitosis are noted.(2a: $\mathrm{x} 40, \mathrm{H} \& \mathrm{E})$. The tumor cells are immunopositive for CD20(2b),CD10.MUM 1(2c,2d) and BCL-6(2e) while immunonegative for CD3(Fig 2f)

lymphadenopathy, no hepatic or splenic involvement, no mediastinal nodal enlargement on chest radiograph and normal white cell count ${ }^{4}$.Clues to help distinguish PPL from pancreatic adenocarcinoma on imaging include a lack of pancreatic duct dilation despite ductal invasion, lymph node involvement below the renal veins and the presence of a bulky homogeneous tumor with no alteration to the Wirsung duct or peri-pancreatic vessels ${ }^{7}$. The first choice of treatment should be a combination of chemotherapy and radiotherapy rather than surgery. With the availability of non-operative diagnostic methods, surgery can be avoided, and improvement in the treatment outcome can also be achieved with combined modality therapy with chemotherapy and radiotherapy.

\section{RICHA MADHAWI ${ }^{1}$ \\ JASPREET KAUR ${ }^{1}$ \\ DEVAJIT NATH ${ }^{2}$ \\ AMIT K DINDA $^{2}$ \\ SUMAN BHASKAR ${ }^{1}$ \\ BIDHU K MOHANTI ${ }^{3}$}

Correspondence: Jaspreet Kaur, ${ }^{1}$ Department of Radiation Oncology,

${ }^{2}$ Department of Pathology,

All India Institute of Medical Sciences, New Delhi, India.

${ }^{3}$ Department of Radiation Oncology,

Fortis Memorial Research Institute,
Gurgaon, Haryana, India. Email:drjaspreet.ro@gmail.com

\section{References}

1. Baylor SM, Berg JW. Cross-classification and survival characteristics of 5,000 cases of cancer of the pancreas. J Surg Oncol. 1973;5:335-8.

2. Koniaris LG, Lillemoe KD, Yeo CJ, Abrams RA, Colemann J,Nakeeb A et al. Is there a role for surgical resection in the treatment of early stage pancreatic lymphoma? J Am Coll Surg. 2000;190:319-30.

3. Lin H, Li SD, Hu XG, Li ZS. Primary pancreatic lymphoma: Report of six cases. World J Gastroenterol. 2006;12:5064-7.

4. Dawson IM, Cornes JS, Morson BC. Primary malignant lymphoid tumors of the intestinal tract: report of 37 cases with a study of factors influencing prognosis. Br J Surg. 1961;49:80-9.

5. Mansour GM, Cucchiaro G, Niotis MT, Fetter BF, Moore J, Rice RR. Surgical management of pancreatic lymphoma. Arch Surg. 1989;124:1287-9.

6. Salvatore JR, Cooper B, Shah I, Kummet T. Primary pancreatic lymphoma: a case report, literature review and proposal for nomenclature. Med Oncol. 2000;17:237-47.

7. Merkle EM, Bender GN, Brambs HJ. Imaging findings in pancreatic lymphoma: differential aspects. AJR Am J Roentgenol. 2000;174:671-5. 